\title{
Review of: "Identification of the Transcription Factors of CD200R1 and Its Potential Roles in Parkinson's Disease"
}

Abbas Amini

Potential competing interests: The author(s) declared that no potential competing interests exist.

Dear Dr. Bedogni,

The paper entitled "Identification of the Transcription Factors of CD200R1 and Its Potential Roles in Parkinson's Disease" was reviewed. It is a compromising paper elucidating the regulation of CD200R. Due to the importance of this gene in immuno-regulating the CNS and considering the cases studied in this article, it has the necessary criteria for publishing and could be accepted.

Sincerely,

Abbas 